\title{
Multi-Objective Resource Allocation Scheme for D2D Multicast with QoS Guarantees in Cellular Networks
}

\author{
Fangmin $\mathrm{Li}^{1,2, *}$, Yong Zhang ${ }^{2}$ and Mohammed Abdulaziz Aide Al-Qaness ${ }^{2}$ \\ 1 Department of Mathematics and Computer Science, Changsha University, Changsha 410022, China \\ 2 School of Information Engineering, Wuhan University of Technology, Wuhan 430070, China; \\ zhang_yong@whut.edu.cn (Y.Z.); alqaness@whut.edu.cn (M.A.A.A.-Q.) \\ * Correspondence: lifangmin@whut.edu.cn; Tel.: +86-27-8729-0335
}

Academic Editor: Christos Verikoukis

Received: 27 August 2016; Accepted: 19 September 2016; Published: 24 September 2016

\begin{abstract}
Device-to-device (D2D) multicast communication can greatly improve the spectrum utilization in a content delivery scenario. However, the co-channel interference and power consumption brought by D2D bring new challenges. All the D2D multicast groups expect to achieve a higher system capacity with less extra energy cost. In this paper, we investigate the uplink resource allocation issue when D2D multicast groups share the resources with other cellular uses (CUs), while guaranteeing a certain level of quality of service (QoS) to CUs and D2D users. Firstly we address a flexible tradeoff framework in which the system power consumption and the system capacity (i.e., the number of admitted D2D links) are assigned with different weight factors so that these two objectives are jointly considered. Then we propose an efficient resource optimization scheme, which comprises sub-channel allocation and signal-to-interference- plus-noise ratio (SINR) assignment. Numerical results validate the effectiveness of the proposed framework, and demonstrate the advantages in dealing with the proposed multi-objective optimization problem.
\end{abstract}

Keywords: cellular network; multiple device-to-device (D2D) communication; uplink resource sharing; sub-channel allocation

\section{Introduction}

In recent years, the popularity of mobile multimedia services has led to an explosive growth of mobile data traffic. To meet various requirements for local wireless services, such as mobile TV, teleconferencing, multiplayer games, the Third Generation Partnership Project (3GPP) is focusing on Device-to-Device (D2D) communication as a goal in Long Term Evolution-Advanced (LTE-A) [1]. With the support of evolved node base stations (eNBs) in a cellular network, D2D communication provides direct data transmission among user equipments (UEs). The proximity of D2D communication brings many benefits for conventional cellular networks, such as high transmission rates, low delays, extended cell coverage and so on [2,3].

As a promising technique of future cellular network, D2D communication is being intensively mined for its potentials of wireless application by worldwide researchers. The authors in [4] propose a social-aware D2D MAC protocol that exploits social network properties of community to reduce the energy consumption through D2D collaboration and D2D relaying. Depending on how the cellular spectrum used by UEs, different resource sharing modes of D2D communication have been investigated, including non-orthogonal mode (i.e., underlay D2D communication) [5-7], orthogonal mode (overlay D2D communication) [8] and outband mode D2D communication [9]. Among these, underlay D2D communication can improve the spectrum efficiency by allocating the same resources to D2D users and cellular users (CUs), however, co-channel interference is introduced in this case, and thus careful resource allocation is required for coexistence of CUs and D2D users. Most of 
the existing research on D2D resource allocation concerns more about D2D unicast communication scenario [10-13]. In [10], an alternative greedy heuristic algorithm is proposed to solve the mixed integer non-linear programing (MINLP) resource allocation problem, which can lessen interference to the primary cellular network utilizing channel gain information. To improve the performance of D2D UE, a proportional fair scheduling algorithm is proposed in [11] for D2D users sharing resources with CUs. In [12], a low complexity power control algorithm is proposed to avoid the interference issue in the case of one D2D pair sharing sub-channel with one CU. The joint subchannel assignment and power control design using dynamic spectrum sharing is conducted in [13], where the target is maximizing the sum-rate while guaranteeing minimum individual cellular link data rates. The method of game theory has also been investigated and introduced into resource allocation for D2D communication in several existing works [14-16]. In [14], a coalition game approach is employed to address the D2D resource sharing that aim at maximizing the energy efficiency of overall system. Both works [15] and [16] employ the non-cooperative game for D2D content dissemination scenarios to achieve transmission delay reducing as well as energy efficiency increasing.

Only a limited amount of the previous work take into consideration the resource management for D2D multicast communication. Because of the difference of data rate among multiple D2D links in multicast communication, the capacity of multicast group is restricted to the worst channel condition. An interference coordination scheme for D2D multicast is proposed in [17], which proposes an efficient power control scheme to obtain an upper bound on D2D transmit power and based on that power level, a suboptimal resource block allocation scheme is proposed. However, this scheme focus on only a single D2D multicast group sharing resources with multiple CUs. The authors of $[18,19]$ consider the content delivery scenario of D2D multicast. In [18], a model where UEs cooperate on the short range by forming coalitions is proposed to improve energy efficiency, while the QoS of UE is not considered. A practical multicast clustering and power controlling strategy with theoretical analysis using game theory is proposed in [19] for the target of decreasing the data distribution time and achieving the QoS requirement of requested uses. However, cellular channel allocation is ignored in this scheme. The authors in [20] present a joint power and channel allocation algorithm to maximize the overall throughput while preserving certain level of QoS for both CUs and D2D groups. However, in this research, each D2D group can reuse the spectrum resource of at most one CU.

This paper focus on the radio resource allocation problem for D2D multicast communications in cellular networks, where one multicast group can reuse multiple cellular spectrum resources. In this case of content dissemination application, the data requests from all UE should be satisfied as much as possible. Nevertheless, with the increase in the number of admitted D2D links, the power consumption of D2D transmitter becomes higher and cannot be ignored. Therefore, optimizing multiple objectives at the same time has a more practical significance in the resource allocation problem. As an important tool, multi-objective optimization has been studied in several recent works. The authors in [21] propose a robust resource allocation strategy to maximize energy harvesting efficiency while minimizing total transmit power in a cognitive network. Similarly, A. multiobjective framework is proposed in [22] to simultaneously improve the network energy efficiency and system throughput. Inspired by these studies, we employ a new multiple-objective optimization framework with the aim to jointly minimize the power consumption of D2D communication and maximize the system capacity of D2D groups. In particular, the main contributions of our work are as follows: (1) We formulate a simple but flexible multi-objective optimization framework via introducing weighted factors for power consumption and system capacity. In addition, we consider a certain level of SINR to guarantee the QoS requirements for both CUs and D2D groups. The framework allows network operators to adjust the tradeoff between the two objectives with various preferences; (2) We use the way of one D2D group sharing sub-channels with multiple CUs to allocate cellular resources, which further enhances the performance of our optimization; (3) We develop an efficient resource allocation scheme to achieve the desired tradeoff for given preferences (i.e., coefficients for power consumption and system capacity). 
The remaining of the paper is organized as follows. In Section 2, we describe the system model and problem optimization. In Section 3, we derive and propose the resource allocation algorithm for D2D multicast communication. Simulation results are shown and discussed in Section 4. Finally, conclusion is drawn in Section 5.

\section{System Model and Problem Formulation}

\subsection{System Model}

We consider a single cellular system supporting D2D communication where $M$ D2D multicast groups coexist with $K$ CUs, as depicted in Figure 1 . Let $\mathcal{K}=\{1,2, \ldots, K\}$ and $\mathcal{M}=\{1,2, \ldots, M\}$ denote the index sets of CUs and D2D groups respectively. The available spectrum is divided into $K$ orthogonal sub-channels, and each of them has been pre-assigned to a CU. Besides, $M$ D2D groups are trying to access into the cellular system in non-orthogonal spectrum sharing model. We adopt uplink resource sharing of cellular network, which is more effective than downlink resource sharing in a fully loaded cellular network, as demonstrated in [23]. We assume in this paper a single multicast group can share spectrum resources with multiple CUs and each CU's spectrum resource can only be reused by at most one D2D group in order to avoid cross-interference between different D2D groups. Additionally, we assume the eNB can obtain the perfect channel state information (CSI) of all the links, based on which, the available spectrum resources are allocated effectively to the CUs and the D2D groups.

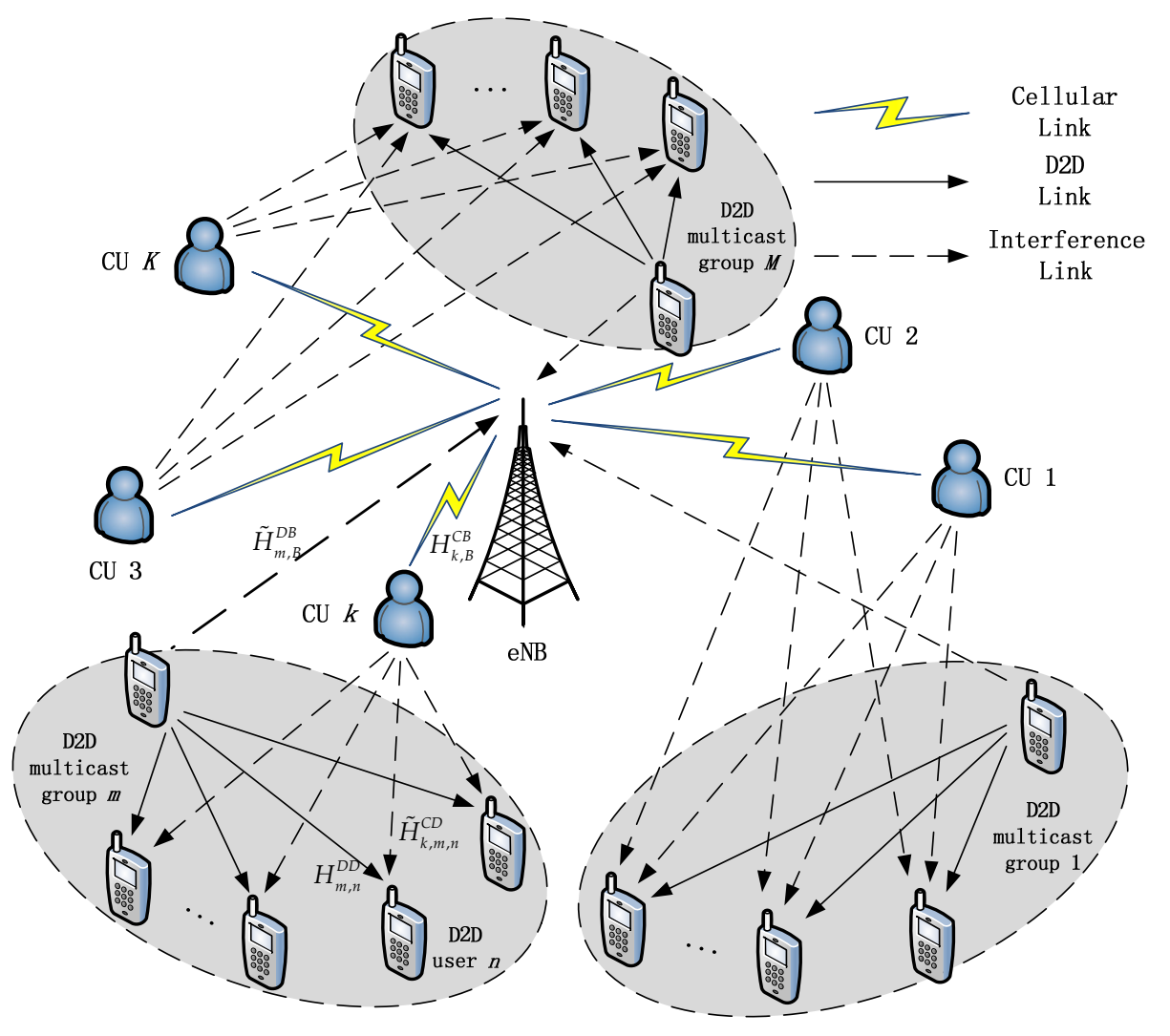

Figure 1. System model of Device-to-device (D2D) multicast groups and cellular uses (CUs).

Define a set of binary variables $\omega_{m, k}$ with $\omega_{m, k}=1$ if the $m$-th D2D group reuses channel $k$ and $\omega_{m, k}=0$ otherwise. For $\mathrm{CU} k \in \mathcal{K}$, the maximum transmission power is limited to $p_{k}^{C}$. The signal to interference plus noise ratio (SINR) of eNB on the sub-channel $k$ can be expressed as 


$$
\gamma_{k}^{C}=\frac{p_{k}^{C} H_{k, B}^{C B}}{\sum_{m \in \mathcal{M}} \omega_{m, k} p_{m, k}^{D} \widetilde{H}_{m, B}^{D B}+\sigma_{0}^{2}},
$$

where $H_{k, B}^{C B}$ and $\widetilde{H}_{m, B}^{D B}$ represent channel gain of the cellular communication link from CU $k$ to the eNB and the interference link from D2D transmitter $m$ to the eNB, respectively. $p_{k}^{C}$ and $p_{m, k}^{D}$ represent the transmission powers of the $\mathrm{CU} k$ and the $m$-th D2D group transmitter on sub-channel $k$, respectively. $\sigma_{0}^{2}$ represents the noise power on the sub-channel $k$. For simplicity, we assume that the noise power is the same for the CUs and the D2D links on each sub-channel.

Let $\mathcal{D}_{m}$ represent the set of D2D receivers in the $m$-th multicast group. Then, $\left|\mathcal{D}_{m}\right|$ is the total number of receivers in the group, where $|\cdot|$ is to derive the cardinality of the corresponding set. The SINR of D2D receiver $n \in \mathcal{D}_{m}$ in the $m$-th multicast group on sub-channel $k$ can be expressed as

$$
\gamma_{n, m, k}^{D}=\frac{p_{m, k}^{D} H_{m, n}^{D D}}{p_{k}^{C} \widetilde{H}_{k, m, n}^{C D}+\sigma_{0}^{2}}
$$

where $H_{m, n}^{D D}$ and $\widetilde{H}_{k, m, n}^{C D}$ represent channel gain of the cellular communication link from the $m$-th D2D group transmitter to its desired D2D receiver $n$ and the interference link from CU $k$ to D2D receiver $n$ in group $m$.

For the D2D link between multicast transmitter $m$ and receiver $n \in \mathcal{D}_{m}$, we define its channel quality on the sub-channel $k$ by

$$
q_{n, m, k}^{D}=\frac{H_{m, n}^{D D}}{p_{k}^{C} \widetilde{H}_{k, m, n}^{C D}+\sigma_{0}^{2}} .
$$

In order to minimize the D2D link's power consumption, there is no need to transmit data in minimum rate to ensure all D2D users receive data correctly. Let $q_{n, m, k}^{D}$ represent the lowest channel quality of D2D links in $m$-th group on sub-channel $k$, the set of admitted D2D receivers in $m$-th multicast group on sub-channel $k$ whose channel quality is higher than user $n$ can be expressed as

$$
A_{n, m, k}=\left\{n^{\prime} \in D_{m} \mid q_{n^{\prime}, m, k}^{D} \geq q_{n, m, k}^{D}\right\} .
$$

Furthermore, $X_{n, m, k}=\left|A_{n, m, k}\right|$ shows the number of D2D links that are admitted to transmit data correctly in $m$-th D2D group on sub-channel $k$.

\subsection{Problem Formulation}

In this paper, our goal is to gain minimal D2D power consumption with maximal system capacity while satisfying the QoS requirement of CUs and D2D users. Hence, we introduce weighted coefficients for power and capacity to strike and regulate the balance between D2D links' aggregated power consumption minimization and system capacity maximization. For simplicity, we denote the SINR thresholds of cellular links and D2D links as $\Gamma_{k}^{C}$ and $\Gamma_{m}^{D}$, which must be attained to meet the individual QoS requirement. In addition, we use $\mathcal{S}_{m}=\left\{k \in \mathcal{K} \mid \omega_{m, k}=1\right\}$ to denote the set of the sub-channels which are assigned to the $m$-th multicast group. Then, by using the linear weighted aggregative method, we formulate the multi-objective optimization problem as

$$
\min _{\left\{p_{k}^{C}, p_{m, k^{\prime}}^{D} \omega_{m, k}\right\}_{m \in \mathcal{M}}} \sum_{k \in \mathcal{K}} \omega_{m, k}\left\{\frac{\eta \cdot p_{m, k}^{D}}{P_{m, k, \max }^{D}}-\frac{(1-\eta) \cdot X_{n, m, k}}{\left|\mathcal{D}_{m}\right|}\right\},
$$




$$
\begin{aligned}
& \text { s.t. } \quad \mathrm{C} 1: \sum_{k \in \mathcal{K}} \omega_{m, k} \log _{2}\left(1+\gamma_{n, m, k}^{D}\right) \geq \log _{2}\left(1+\Gamma_{m}^{D}\right) \text {, } \\
& \forall m \in \mathcal{M}, n \in \mathcal{D}_{m}, \\
& \text { C2 : } \gamma_{k}^{C} \geq \Gamma_{k}^{C}, \forall k \in \mathcal{K} \text {, } \\
& \text { C3: } 0 \leq \sum_{k \in \mathcal{K}} \omega_{m, k} p_{m, k}^{D} \leq P_{t h r}^{D}, \forall m \in \mathcal{M} \text {, } \\
& \text { C4 : } 0 \leq p_{k}^{C} \leq P_{t h r}^{C}, \forall k \in \mathcal{K}, \\
& \text { C5 : } \omega_{m, k} \in\{0,1\}, \forall k \in \mathcal{K}, m \in \mathcal{M} \text {, } \\
& \text { C6 : } \sum_{k \in \mathcal{K}} \omega_{m, k}=\left|\mathcal{S}_{m}\right|, \forall m \in \mathcal{M} \text {. } \\
& \text { C7 : } \sum_{m \in \mathcal{M}} \omega_{m, k} \leq 1, \forall k \in \mathcal{K} \text {. }
\end{aligned}
$$

where $P_{m, k, \max }^{D}$ represents the maximum expected power of D2D group $m$. The positive coefficients $\eta$ and $1-\eta$ regulate the tradeoff between power consumption and system capacity, where $\eta \in(0,1)$. $\mathrm{C} 1$ and C2 stand for the QoS guarantees for the D2D links and cellular links. C3 and C4 are the transmission power constraints for D2D groups and CUs. C5 and C6 indicate each channel can be reused by at most one $\mathrm{D} 2 \mathrm{D}$ group.

\section{The Proposed Resource Allocation for D2D Communication}

Obviously, the optimization problem (5) in an MINLP problem which is a NP-hard problem and hard to get an optimal and satisfactory solution, as the complexity may increase exponentially with the problem size. Next, we propose an effective source allocation algorithm to address this problem.

\subsection{Problem Equivalence}

Consider CU $k \in \mathcal{K}$, D2D multicast group $m \in \mathcal{M}$ and D2D receiver $n \in \mathcal{D}_{m}$. If the frequency resource of $C U k$ is reused by D2D multicast group $m$, i.e., $\omega_{m, k}=1$, Equation (1) can be rewritten as

$$
\gamma_{k, m}^{C}=\frac{p_{k}^{C} H_{k, B}^{C B}}{p_{m, k}^{D} \widetilde{H}_{m, B}^{D B}+\sigma_{0}^{2}} .
$$

According to Equation (6) and constraint $\mathrm{C} 2$ and $\mathrm{C} 4$ in Equation (5), $P_{m, k}^{D}$ reaches a maximum value when $\gamma_{k}^{C}=\Gamma_{k}^{C}$ and $p_{k}^{C}=P_{t h r}^{C}$ are both satisfied. Substituting $\gamma_{k}^{C}=\Gamma_{k}^{C}$ and $p_{k}^{C}=P_{t h r}^{C}$ into Equation (6), we can get

$$
P_{m, k, \max }^{D}=\min \left\{\frac{P_{t h r}^{C} H_{k, B}^{C B}}{\widetilde{H}_{m, B}^{D B} \Gamma_{k}^{C}}-\frac{\sigma_{0}^{2}}{\widetilde{H}_{m, B}^{D B}}, P_{t h r}^{D}\right\} .
$$

In addition, by combining Equations (2) and (6), the transmission power of D2D multicast transmitter $\mathrm{m}$ and $\mathrm{CU} k$ can be obtained as follows:

$$
\left\{\begin{array}{l}
p_{m, k}^{D}\left(\gamma_{k, m}^{C} \gamma_{n, m, k}^{D}\right)=\frac{\left(H_{k, B}^{C B}+\widetilde{H}_{k, m, n}^{C D} \gamma_{k, m}^{C}\right) \gamma_{n, m, k}^{D} \sigma_{0}^{2}}{H_{k, B}^{C B} H_{m, n}^{D D}-\widetilde{H}_{m, B}^{D B} \widetilde{H}_{k, m, n}^{C D} \gamma_{k, m}^{C} \gamma_{n, m, k}^{D}} \\
p_{k}^{C}\left(\gamma_{k, m}^{C}, \gamma_{n, m, k}^{D}\right)=\frac{\left(H_{m, n}^{D D}+\widetilde{H}_{k, m, n}^{C D} \gamma_{n, m, k}^{D}\right) \gamma_{k, m}^{C} \sigma_{0}^{2}}{H_{k, B}^{C B} H_{m, n}^{D D}-\widetilde{H}_{m, B}^{D B} \widetilde{H}_{k, m, n}^{C D} \gamma_{k, m}^{C} \gamma_{n, m, k}^{D}}
\end{array}\right.
$$

From Equation (8), we can observe that $P_{m, k}^{D}$ and $p_{k}^{C}$ are monotonically increasing in $\gamma_{k, m}^{C}$ for fixed $\gamma_{n, m, k}^{D}$. In order to minimize the power consumption of D2D group, we need to keep the minimum QoS guarantees of cellular link, i.e., $\gamma_{k, m}^{C *}=\Gamma_{k}^{C}$. Substituting $\gamma_{k, m}^{C *}=\Gamma_{k}^{C}$ into Equation (8) leads to 


$$
\left\{\begin{array}{c}
p_{m, k}^{D}\left(\gamma_{k, m}^{C *}, \gamma_{n, m, k}^{D}\right)=\frac{\left(H_{k, B}^{C B}+\widetilde{H}_{k, m, n}^{C D} \Gamma_{k}^{C}\right) \gamma_{n, m, k}^{D} \sigma_{0}^{2}}{H_{k, B}^{C B} H_{m, n}^{D D}-\widetilde{H}_{m, B}^{D B} \widetilde{H}_{k, m, n}^{C D} \Gamma_{k}^{C} \gamma_{n, m, k}^{D}} \\
p_{k}^{C}\left(\gamma_{k, m}^{C *}, \gamma_{n, m, k}^{D}\right)=\frac{\left(H_{m, n}^{D D}+\widetilde{H}_{k, m, n}^{C D} \gamma_{n, m, k}^{D}\right) \Gamma_{k}^{C} \sigma_{0}^{2}}{H_{k, B}^{C B} H_{m, n}^{D D}-\widetilde{H}_{m, B}^{D B} \widetilde{H}_{k, m, n}^{C D} \Gamma_{k}^{C} \gamma_{n, m, k}^{D}}
\end{array}\right.
$$

Then, we substitute $p_{m, k}^{D}\left(\gamma_{k, m}^{C *}, \gamma_{n, m, k}^{D}\right)$ into Equation (5), and the optimization problem can be rewritten as

$$
\min _{\left\{\gamma_{n, m, k^{\prime}}^{D} \boldsymbol{\omega}_{m, k}\right\}_{m \in \mathcal{M}}} \sum_{m \in \mathcal{K}} \omega_{m, k}\left\{\eta \frac{p_{m, k}^{D}\left(\gamma_{k, m}^{C *} \gamma_{n, m, k}^{D}\right)}{P_{m, k, \max }^{D}}-(1-\eta) \frac{X_{n, m, k}}{\left|\mathcal{D}_{m}\right|}\right\}
$$

Define

$$
\Phi\left(\gamma_{k, m}^{C *}, \gamma_{n, m, k}^{D}\right)=\eta \frac{p_{m, k}^{D}\left(\gamma_{k, m}^{C *}, \gamma_{n, m, k}^{D}\right)}{P_{m, k, \max }^{D}}-(1-\eta) \frac{X_{n, m, k}}{\left|\mathcal{D}_{m}\right|} .
$$

Then Eqaution (10) can be expressed as

$$
\min _{\left\{\gamma_{n, m, k^{\prime}}^{D} \omega_{m, k}\right\}_{m \in \mathcal{M}}} \sum_{k \in \mathcal{K}} \omega_{m, k} \Phi\left(\gamma_{k, m}^{C *}, \gamma_{n, m, k}^{D}\right) .
$$

As explained above, if the resource of CU $k$ is reused by D2D group $m$, the constraints $C 5, C 6$ and C7 should be satisfied. Then, we introduce the following Proposition.

Proposition 1. The optimal solutions of the power values $p_{m, k}^{D}$ of the optimization problem in Equation (12) must be the optimal solutions of the optimization problem $\min _{\left\{\gamma_{n, m, k}^{D}\right\} k \in \mathcal{S}_{m}} \Phi\left(\gamma_{k, m}^{C *}, \gamma_{n, m, k}^{D}\right)$.

Proof. The proof is presented in Appendix A.

It can be seen from Proposition 1 that the original dimensional non-convex optimization problem can be transformed into two separate optimization sub-problems. In the first sub-problem, for single D2D group with a given multiple sub-channels reusing, we find the optimal SINR value on each sub-channel to achieve the multi-objective optimization. In the second sub-problem, for multiple D2D groups sharing the resources with multiple CUs, we propose a practice algorithm to allocate each sub-channel to the D2D multicast groups.

\subsection{SINR Assignment for One D2D Multicast Group}

Without loss of generality, we consider the $m$-th D2D group, which reuses spectrum resources with the CUs specified in $\mathcal{S}_{m}$. According to constraint C4 in Equation (5), if the spectrum resource of CU $k$ is reused by D2D group $m, 0 \leq p_{k}^{C} \leq P_{t h r}^{C}$ should be satisfied. By substituting Equation (9) into constraint $\mathrm{C} 4$, we have

$$
0 \leq \frac{H_{m, n}^{D D}+\widetilde{H}_{k, m, n}^{C D} \gamma_{n, m, k}^{D}}{H_{k, B}^{C B} H_{m, n}^{D D}-\widetilde{H}_{m, B}^{D B} \widetilde{H}_{k, m, n}^{C D} \Gamma_{k}^{C} \gamma_{n, m, k}^{D}} \Gamma_{k}^{C} \sigma_{0}^{2} \leq P_{t h r}^{D} .
$$

Form above inequality, we can get

$$
\left\{\begin{aligned}
& \gamma_{n, m, k}^{D}<\frac{H_{k, B}^{C B} H_{m, n}^{D D}}{\widetilde{H}_{m, B}^{D B} \widetilde{H}_{k, m, n}^{C D} \Gamma_{k}^{C}} \\
& \gamma_{n, m, k}^{D} \leq \frac{P_{t h r}^{D} H_{k, B}^{C B} H_{m, n}^{D D}-H_{m, n}^{D D} \Gamma_{k}^{C} \sigma_{0}^{2}}{\widetilde{H}_{k, m, n}^{C D} \Gamma_{k}^{C}\left(\sigma_{0}^{2}+P_{t h r}^{D} \widetilde{H}_{m, B}^{D B}\right)}
\end{aligned}\right.
$$


Define

$$
\gamma_{n, m, k, \max }^{D}=\min \left\{\frac{H_{k, B}^{C B} H_{m, n}^{D D}}{\tilde{H}_{m, B}^{D B} \tilde{H}_{k, m, n}^{C D} \Gamma_{k}^{C}}, \frac{P_{t h r}^{D} H_{k, B}^{C B} H_{m, n}^{D D}-H_{m, n}^{D D} \Gamma_{k}^{C} \sigma_{0}^{2}}{\tilde{H}_{k, m, n}^{C D} \Gamma_{k}^{C}\left(\sigma_{0}^{2}+P_{t h r}^{D} \tilde{H}_{m, B}^{D B}\right)}\right\} .
$$

According to Proposition 1, the multi-objective optimization model for D2D group $m$ can be simplified to the following problem:

$$
\begin{gathered}
\min _{\left\{\gamma_{n, m, k}^{D}\right\}} \sum_{k \in \mathcal{S}_{m}} \Phi\left(\gamma_{k, m}^{C *}, \gamma_{n, m, k}^{D}\right) \\
\text { s.t. } \quad \mathrm{C} 1: \sum_{k \in \mathcal{S}_{m}} \log _{2}\left(1+\gamma_{n, m, k}^{D}\right) \geq \log _{2}\left(1+\Gamma_{m}^{D}\right), \\
\mathrm{C} 2: 0 \leq \gamma_{n, m, k}^{D} \leq \gamma_{n, m, k, \max ^{\prime}}^{D}, \forall k \in \mathcal{S}_{m} .
\end{gathered}
$$

By taking the second order derivative of the $\Phi$ versus $\gamma_{n, m, k}^{D}$, we can get

$$
\begin{aligned}
\frac{\partial^{2} \Phi}{\partial \gamma_{n, m, k}^{D}{ }^{2}} & =\frac{2 \eta \sigma_{0}^{2} H_{k, B}^{C B} H_{m, n}^{D D} \widetilde{H}_{m, B}^{D B} \widetilde{H}_{k, m, n}^{C D} \Gamma_{k}^{C}}{P_{m, k, \max }^{D}} \cdot \frac{H_{k, B}^{C B}+\widetilde{H}_{k, m, n}^{C D} \Gamma_{k}^{C}}{H_{k, B}^{C B} H_{m, n}^{D D}-\widetilde{H}_{m, B}^{D B} \widetilde{H}_{k, m, n}^{C D} \Gamma_{k}^{C} \gamma_{n, m, k}^{D}}, \\
& >0
\end{aligned}
$$

indicating that $\Phi$ is a convex function. Then, it is obvious that the optimization problem in Equation (16) is a convex optimization problem about $\gamma_{n, m, k}^{D}$ [24].

In order to solve Equation (16), we introduce the non-negative Lagrangian multipliers $\lambda \geq 0$, associating with the constraint $\mathrm{C} 1$ in Equation (5). Then, we write its Lagrangian function as

$$
L\left(\left\{\gamma_{n, m, k}^{D}\right\}, \lambda\right)=\sum_{k \in \mathcal{S}_{m}} \Phi\left(\gamma_{k, m}^{C *}, \gamma_{n, m, k}^{D}\right)+\lambda\left[\log _{2}\left(1+\Gamma_{m}^{D}\right)-\sum_{k \in \mathcal{S}_{m}} \log _{2}\left(1+\gamma_{n, m, k}^{D}\right)\right] .
$$

By applying Karush-Kuhn-Tucker (KKT) conditions [14], the first order optimality conditions and the equation constraint are given as follows,

$$
\begin{aligned}
\frac{\partial L\left(\left\{\gamma_{n, m, k}^{D}\right\}, \lambda\right)}{\partial \gamma_{n, m, k}^{D}}= & \frac{\eta \sigma_{0}^{2} H_{k, B}^{C B} H_{m, n}^{D D}\left(H_{k, B}^{C B}+\widetilde{H}_{k, m, n}^{C D} \Gamma_{k}^{C}\right)}{P_{m, k, \max }^{D}\left(H_{k, B}^{C B} H_{m, n}^{D D}-\widetilde{H}_{m, B}^{D B} \widetilde{H}_{k, m, n}^{C D} \Gamma_{k}^{C} \gamma_{n, m, k}^{D}\right)^{2}}-\frac{\lambda}{\ln 2\left(1+\gamma_{n, m, k}^{D}\right)}, \\
= & 0, \forall k \in \mathcal{S}_{m} \\
& \lambda\left[\log _{2}\left(1+\Gamma_{m}^{D}\right)-\sum_{k \in \mathcal{S}_{m}} \log _{2}\left(1+\gamma_{n, m, k}^{D}\right)\right]=0 .
\end{aligned}
$$

From Equation (19), it can be easily found that the optimal $\lambda$ must be positive, since $\lambda=0$ implies that the equation $\eta \sigma_{0}^{2} H_{k, B}^{C B} H_{m, n}^{D D}\left(H_{k, B}^{C B}+\widetilde{H}_{k, m, n}^{C D} \Gamma_{k}^{C}\right)=0$ is satisfied for all $k \in \mathcal{S}_{m}$, which is impossible in practice. With simple mathematical operations, Equation (19) can be rewritten into following quadratic equation:

$$
A_{k}(\lambda) \gamma_{n, m, k}^{D}{ }^{2}+B_{k}(\lambda) \gamma_{n, m, k}^{D}+C_{k}(\lambda)=0
$$

where 


$$
\begin{aligned}
& A_{k}(\lambda)=\left(\widetilde{H}_{m, B}^{D B} \widetilde{H}_{k, m, n}^{C D} \Gamma_{k}^{C}\right)^{2} \lambda \\
& B_{k}(\lambda)=-2 \lambda H_{k, B}^{C B} H_{m, n}^{D D} \widetilde{H}_{m, B}^{D B} \widetilde{H}_{k, m, n}^{C D} \Gamma_{k}^{C}-D_{k} \\
& C_{k}(\lambda)=\left(H_{k, B}^{C B} H_{m, n}^{D D}\right)^{2} \lambda-D_{k} \\
& D_{k}=\ln 2 \cdot \frac{\eta \sigma_{0}^{2} H_{k, B}^{C B} H_{m, n}^{D D}\left(H_{k, B}^{C B}+\widetilde{H}_{k, m, n}^{C D} \Gamma_{k}^{C}\right)}{P_{m, k, \max }^{D}}
\end{aligned}
$$

Then we can easily get

$$
\begin{aligned}
B_{k}(\lambda)^{2}-4 A_{k}(\lambda) C_{k}(\lambda) & =D_{k}^{2}+4 \lambda D_{k} \widetilde{H}_{m, B}^{D B} \widetilde{H}_{k, m, n}^{C D} \Gamma_{k}^{C}\left(\widetilde{H}_{m, B}^{D B} \widetilde{H}_{k, m, n}^{C D} \Gamma_{k}^{C}+H_{k, B}^{C B} H_{m, n}^{D D}\right) . \\
& >0
\end{aligned}
$$

Therefore, the available solution to Equation (21) can be obtained as follows:

$$
\gamma_{n, m, k}^{D}(\lambda)_{1,2}=\frac{-B_{k}(\lambda) \pm \sqrt{B_{k}(\lambda)^{2}-4 A_{k}(\lambda) C_{k}(\lambda)}}{2 A_{k}(\lambda)} .
$$

From the above equation, we find

$$
\begin{aligned}
\gamma_{n, m, k}^{D}(\lambda)_{1} & =\frac{-B_{k}(\lambda)+\sqrt{B_{k}(\lambda)^{2}-4 A_{k}(\lambda) C_{k}(\lambda)}}{2 A_{k}(\lambda)} \\
& >\frac{-B_{k}(\lambda)}{2 A_{k}(\lambda)}=\frac{2 \lambda H_{k, B}^{C B} H_{m, n}^{D D} \widetilde{H}_{m, B}^{D B} \widetilde{H}_{k, m, n}^{C D} \Gamma_{k}^{C}+D_{k}}{2\left(\widetilde{H}_{m, B}^{D B} \widetilde{H}_{k, m, n}^{C D} \Gamma_{k}^{C}\right)^{2} \lambda}, \\
& >\frac{H_{k, B}^{C B} H_{m, n}^{D D}}{\widetilde{H}_{m, B}^{D B} \widetilde{H}_{k, m, n}^{C D} \Gamma_{k}^{C}},
\end{aligned}
$$

which contradicts Equation (14). Consequently, the optimal solution to Equation (21) is given by

$$
\begin{aligned}
\gamma_{n, m, k}^{D}{ }^{*} & =\gamma_{n, m, k}^{D}(\lambda)_{2} \\
& =\left[\frac{-B_{k}(\lambda)-\sqrt{B_{k}(\lambda)^{2}-4 A_{k}(\lambda) C_{k}(\lambda)}}{2 A_{k}(\lambda)}\right]_{0}^{\gamma_{n, m, k \max }^{D},}
\end{aligned}
$$

where $[x]_{0}^{\gamma_{n, m, k, \max }^{D}}$ represents the projection onto the interval $\left[0, \gamma_{n, m, k, \max }^{D}\right]$. Furthermore, according to Equation (20), the optimal $\gamma_{n, m, k}^{D}{ }^{*}$ must satisfy

$$
\sum_{k \in \mathcal{S}_{m}} \log _{2}\left(1+\gamma_{n, m, k}^{D}{ }^{*}\right)=\log _{2}\left(1+\Gamma_{m}^{D}\right) .
$$

Given that $\gamma_{n, m, k}^{D}{ }^{*}$ in Equation (27) depends on $\lambda$ in a highly nonlinear way, directly computing the optimal $\lambda$ is difficult. Nevertheless, the optimal $\lambda$ can be efficiently found via bisection, for which one needs to know an interval containing the optimal $\lambda$. The following result provides such an interval.

Lemma 1. The optimal $\lambda$ satisfying $\sum_{k \in \mathcal{S}_{m}} \log _{2}\left(1+\gamma_{n, m, k}^{D}{ }^{*}\right)=\log _{2}\left(1+\Gamma_{m}^{D}\right)$ is within $\left[\lambda_{\min }, \lambda_{\max }\right]$ where $\lambda_{\max } \triangleq \max _{\mathcal{S}_{m}}\left\{D_{k} \cdot \frac{1+\gamma_{n, m, k, \max }^{D}}{\left(H_{k, B}^{C B} H_{m, n}^{D D}-\widetilde{H}_{m, B}^{D B} \widetilde{H}_{k, m, n}^{C D} \Gamma_{k}^{C} \gamma_{n, m, k, \max }^{D}\right)^{2}}\right\}$ and $\lambda_{\min } \triangleq \min _{\mathcal{S}_{m}}\left\{\frac{D_{k}}{\left(H_{k, B}^{C B} H_{m, n}^{D D}\right)^{2}}\right\}$. 
Proof. The proof is presented in Appendix B.

\subsection{Sub-Channel Allocation for Multiple D2D Multicast Groups}

Before the SINR allocation, the set $\mathcal{S}_{m}$ of CUs sharing resources with the $m$-th D2D group must be determined through sub-channel allocation for the matching between multiple D2D groups and CUs' sub-channels. In this subsection, the details of the sub-channel allocation are introduced.

At the beginning of the allocation, the eNB collects the location information of all the D2D groups. Then, in order to remove coupling of the sub-channel allocation and the SINR assignment, it is assumed that the D2D group transmitters allocate the same SINR $\hat{\gamma}_{n, m, k}^{D}$ on each sub-channel, i.e., the transmission rate of D2D group $\mathrm{m}$ on the sub-channel $\mathrm{k}$ is $\hat{R}_{m, k}^{D}=\log _{2}\left(1+\Gamma_{m}^{D}\right) /[K / M]$, where $[\cdot]$ is to take the integer portion of the output. Based on Proposition 1, in order to achieve the multi-objective optimization goal while guaranteeing the QoS of D2D groups and CUs, the sub-channel allocation problem can be expressed as

$$
\begin{gathered}
\min _{\left\{\omega_{m, k}\right\}} \sum_{m \in \mathcal{M}} \sum_{k \in \mathcal{K}} \omega_{m, k} \Phi\left(\gamma_{k, m}^{C *}, \hat{\gamma}_{n, m, k}^{D}\right) \\
\text { s.t. } \quad \log _{2}\left(1+\hat{\gamma}_{n, m, k}^{D}\right) \geq R_{m, k}^{D}, \\
\quad \text { C5 C7 in }(5)
\end{gathered},
$$

where the SINR of D2D group $m$ on the sub-channel $k$ can be calculated by $\hat{\gamma}_{n, m, k}^{D}=2^{\hat{R}_{m, k}^{D}}-1$. By substituting $\hat{\gamma}_{n, m, k}^{D}$ into Equation (9), we can get

$$
\left\{\begin{array}{c}
p_{m, k}^{D}\left(\gamma_{k, m}^{C *}, \hat{\gamma}_{n, m, k}^{D}\right)=\frac{\left(H_{k, B}^{C B}+\widetilde{H}_{k, m, n}^{C D} \Gamma_{k}^{C}\right) \hat{\gamma}_{n, m, k}^{D} \sigma_{0}^{2}}{H_{k, B}^{C B} H_{m, n}^{D D}-\widetilde{H}_{m, B}^{D B} \widetilde{H}_{k, m, n}^{C D} \Gamma_{k}^{C} \hat{\gamma}_{n, m, k}^{D}} \\
p_{k}^{C}\left(\gamma_{k, m}^{C *}, \hat{\gamma}_{n, m, k}^{D}\right)=\frac{\left(H_{m, n}^{D D}+\widetilde{H}_{k, m, n}^{C D} \hat{\gamma}_{n, m, k}^{D}\right) \Gamma_{k}^{C} \sigma_{0}^{2}}{H_{k, B}^{C B} H_{m, n}^{D D}-\widetilde{H}_{m, B}^{D B} \widetilde{H}_{k, m, n}^{C D} \Gamma_{k}^{C} \hat{\gamma}_{n, m, k}^{D}}
\end{array}\right.
$$

and the lowest channel quality of D2D links in $m$-th multicast on sub-channel $k$ is

$$
\hat{q}_{n, m, k}^{D}=\frac{\hat{\gamma}_{n, m, k}^{D}}{p_{m, k}^{D}\left(\gamma_{k, m}^{C *}, \hat{\gamma}_{n, m, k}^{D}\right)} .
$$

Then, the value of $\Phi\left(\gamma_{k, m}^{C *}, \hat{\gamma}_{n, m, k}^{D}\right)$ is given by

$$
\Phi\left(\gamma_{k, m}^{C *}, \hat{\gamma}_{n, m, k}^{D}\right)=\eta \frac{p_{m, k}^{D}\left(\gamma_{k, m}^{C *}, \hat{\gamma}_{n, m, k}^{D}\right)}{P_{m, k, \max }^{D}}-(1-\eta) \frac{\hat{X}_{n, m, k}}{\left|\mathcal{D}_{m}\right|},
$$

where $\hat{X}_{n, m, k}$ can be calculated by the following formula

$$
\hat{X}_{n, m, k}=\left|\left\{n^{\prime} \in \mathcal{D}_{m} \mid \hat{q}_{n^{\prime}, m, k}^{D} \geq \hat{q}_{n, m, k}^{D}\right\}\right| .
$$

Note that the multi-objective optimization value brought by any D2D multicast group reusing any sub-channel can be roughly measured by $\Phi\left(\gamma_{k, m}^{C *}, \hat{\gamma}_{n, m, k}^{D}\right)$. Therefore, the D2D pair that has the minimum $\Phi\left(\gamma_{k, m}^{C *}, \hat{\gamma}_{n, m, k}^{D}\right)$ has the priority to reuse the $k$-th sub-channel. When the $k$-th sub-channel is allocated to the D2D group $m$, it will be recorded into set $\mathcal{S}_{m}, m=1,2, \ldots, M$ and not be used by other D2D groups. For purpose of fairness among D2D multicast groups, the sub-channels are firstly assigned to D2D groups who meet $\left|\mathcal{S}_{m}\right| \leq \frac{K}{M}-1$. The sub-channel allocation procedure ends up until all sub-channels have been assigned. 


\subsection{Overall Mechanism}

The overall mechanism includes the above two phases to jointly allocate sub-channel and SINR. The first phase described in Section 3.3 is to match multiple sub-channels to D2D multicast groups. After the sub-channel allocation in phase 1 is finished, the SINR assignment method in Section 3.2 is performed to determine the optimal SINR of all D2D links for multi-objective optimization on any given sub-channel from phase 1, the pseudo code of overall algorithm is shown in Algorithm 1.

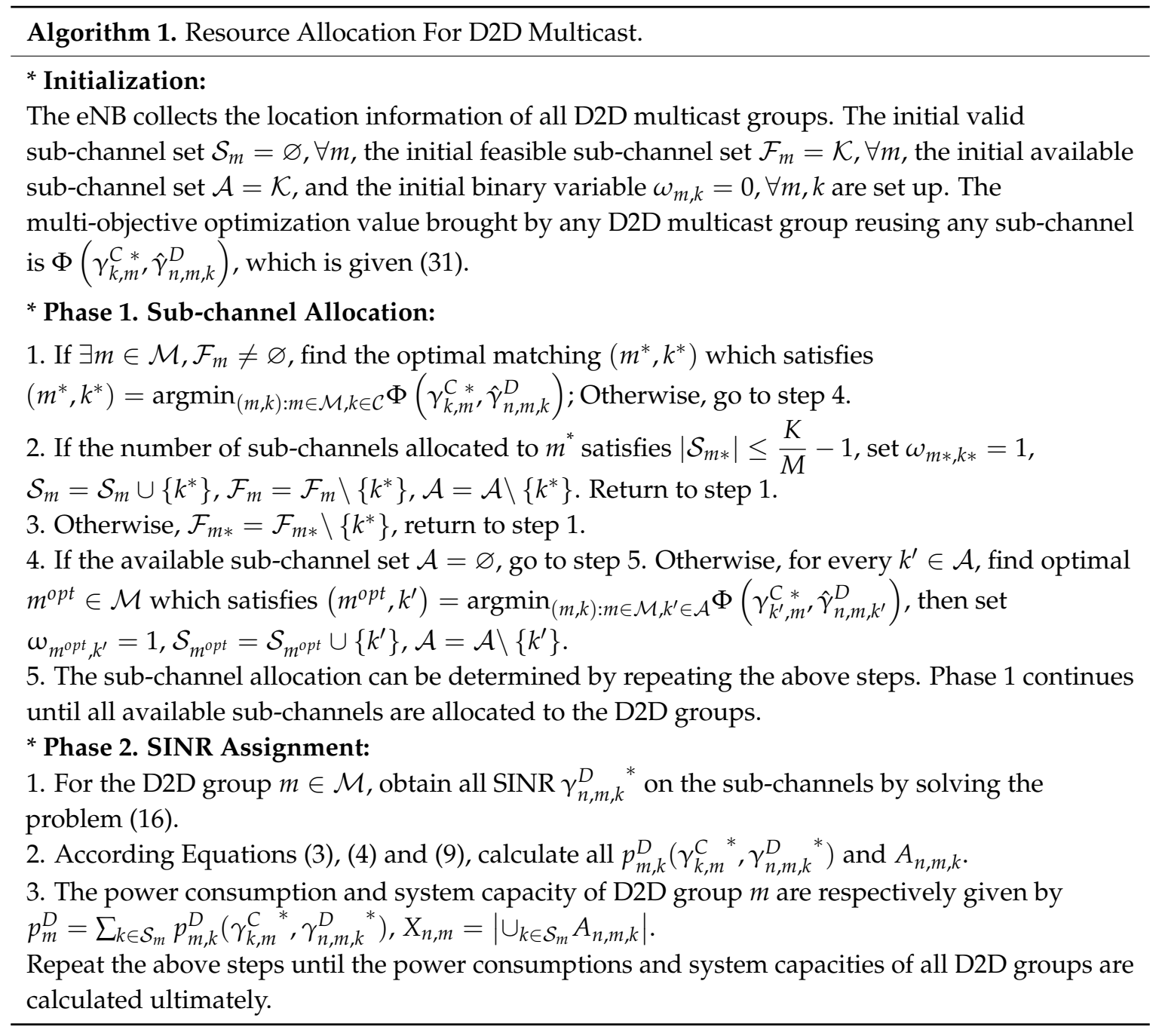

We analyze the complexity of our proposed algorithm in this subsection. In phase 1, we search for the optimal matching of sub-channel resources between all D2D groups and CUs, and then all available sub-channels are allocated to the D2D groups. Hence the complexity of the sub-channel allocation is $\mathcal{O}(M K)$. In phase 2, with $\tau$ as a small acceptable tolerance for bisection algorithm, the complexity of the SINR assignment is $\mathcal{O}\left(K \log _{2}(1 / \tau)\right)$. Therefore the complexity of multi-object optimization resource allocation is $\mathcal{O}(M K)+\mathcal{O}\left(K \log _{2}(1 / \tau)\right)$. Obviously, our algorithm has polynomial complexity which has a linear relation with the scale of $M$ and $K$.

\section{Numerical Results}

In this section, we provide several simulation results to evaluate the performance of our proposed resource allocation scheme. The simulated network is contained in a single cell with a radius of $500 \mathrm{~m}$ where CUs and D2D multicast groups are uniformly and randomly distributed. We follow the clustered distribution model in [25], where D2D multicast groups of radius $r$ are randomly located 
in a cell and the D2D users in each group are randomly distributed in the corresponding cluster. Simulation parameters such as path loss models, maximum transmission power, etc. are the same as those proposed in [26], and the main simulation parameters are listed in Table 1.

Table 1. Main Simulation Parameters.

\begin{tabular}{cc}
\hline Parameter & Value \\
\hline Cellular layout & one isolated cellular cell \\
Cell radius, $R$ & $300 \mathrm{~m} \sim 700 \mathrm{~m}$ \\
Uplink bandwidth & $180 \mathrm{kHz}$ \\
Noise spectral density & $-174 \mathrm{dBm} / \mathrm{Hz}$ \\
Path loss for cellular links & $128.1+37.6 \log 10(\mathrm{~d}(\mathrm{~km}))$ \\
Path loss for D2D links & $148+40 \log 10(\mathrm{~d}(\mathrm{~km}))$ \\
Shadowing standard deviation & $10 \mathrm{~dB}$ for cellular links \\
Noise spectral density & $12 \mathrm{~dB}$ for D2D links \\
Maximum transmission power of CU & $-174 \mathrm{dBm} / \mathrm{Hz}$ \\
Maximum transmission power of D2D & $20 \mathrm{dBm}$ \\
SINR threshold of CU & $20 \mathrm{dBm}$ \\
SINR threshold of CU link & $0 \mathrm{~dB} \sim 10 \mathrm{~dB}$ \\
Number of CUs, $K$ & $10 \mathrm{~dB} \sim 35 \mathrm{~dB}$ \\
Number of D2D groups, $M$ & 20 \\
Number of D2D receivers in each group & $3 \sim 10$ \\
D2D group size, $r$ & 10 \\
\hline
\end{tabular}

We evaluate the performance of the proposed scheme by comparing it with four benchmarks: (1) power minimization ("MinP"); (2) capacity maximization ("MaxC"); (3) multi-objective optimization with each D2D group reusing one single sub-channel ("Single"); and (4) sum rate Maximization scheme in [12]. The first three can be regarded as the special cases of the proposed scheme by setting $(\eta=1)$, $(\eta=0)$ and $\left(\left|\mathcal{S}_{m}\right|=1\right)$ for "MinP", "MaxC" and "Single". The fourth one is a pure throughput optimization scheme, which assumes each D2D group can reuse the sub-channel of at most one CU.

Figure 2 presents the power consumption and system capacity performance with the varying of the weighted factor $\eta$, where $K=20, M=10,\left|\mathcal{D}_{m}\right|=10, R=500 \mathrm{~m}, r=50 \mathrm{~m}, \Gamma_{k}^{C}=5 \mathrm{~dB}$ and $\Gamma_{m}^{D}=35 \mathrm{~dB}$. For the proposed scheme, the power consumption preference is enhanced with larger $\eta$, while, from Figure $2 b$, the system capacity preference requires smaller $\eta$ with upper bound identical to the performance of the benchmark "MaxC". Hence, using the proposed scheme, the operator can flexibly configure the network with different preferences to satisfy specific system or user requirements simply by varying the weighted factors. Furthermore, it can be seen from Figure 2 that for the proposed scheme, increasing $\eta$ decreases its power consumption value from 150 to 82 , and its number of admitted D2D links from 87.3 to 76.4; while the power consumption and the number of admitted D2D links for "MaxR" are about 461.8 and 43.5, respectively. This implies that compared to the "MaxR" scheme, the proposed scheme seems to achieve more admitted D2D links in the network by consuming less power. This is because adopting the single sub-channel reusing for the "MaxR" scheme makes the power on the sub-channel fixed while the proposed scheme enables one D2D group to reuse multiple sub-channels and fully explore the spatial gain among different sub-channels, and be able to adjust power level on each sub-channel so as to perform power consumption minimization as well as admitting the maximum number of D2D links into the system. Moreover, we observe that the performance of the "Single" scheme is close to "MaxR" when weighted factor $\eta \leq 0.2$, while the consumed power in the "Single" scheme is less than in "MaxR" at the cost of removing more D2D links from the system. 


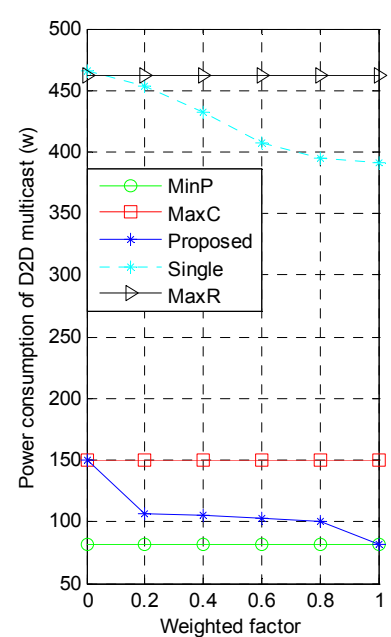

(a)

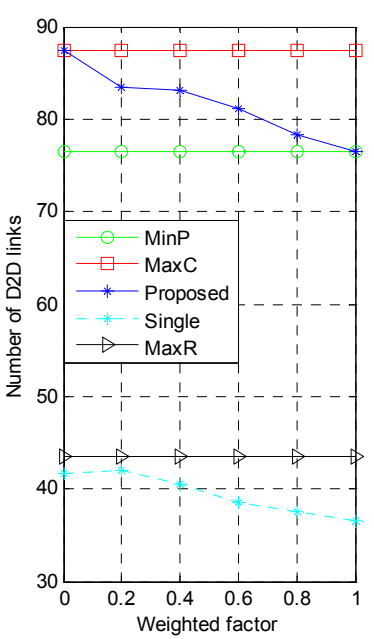

(b)

Figure 2. Power consumption and number of D2D links versus weighted factor: (a) Impact of weighted factor on power consumption; (b) Impact of weighted factor on system capacity.

Figure 3 shows the power consumption and system capacity with different D2D group size, where $K=20, M=10,\left|\mathcal{D}_{m}\right|=10, R=500 \mathrm{~m}, \Gamma_{k}^{C}=5 \mathrm{~dB}, \Gamma_{m}^{D}=35 \mathrm{~dB}$ and $\eta=0.5$. As shown in Figure 3b, the number of admitted D2D links decreases with increasing of the D2D group size. Especially, the "MaxR" and "Single" schemes fall faster than the proposed scheme. Since the channel gain of D2D link decreases when the multicast radius increases, more D2D links are likely to be removed to benefit the QoS maintenance of the remaining system, with bigger influence on the single sub-channel reusing scheme ("MaxR" and "Single"). However, the change in power consumption is not monotonic and depends on the following two factors: (i) more transmission power is required for the D2D groups to satisfy the SINR threshold constraint; and (ii) the worst channel condition of D2D multicast becomes relatively better along with the removed D2D links, thus the required transmission power decreases. It is seen from Figure 3a that the power consumption of "MaxR" shows a trend of earlier increase and later decrease. The decrement of proposed scheme in the number of admitted D2D links is much less than that of the "MaxR" scheme, thus power consumption of the proposed scheme keeps growing.

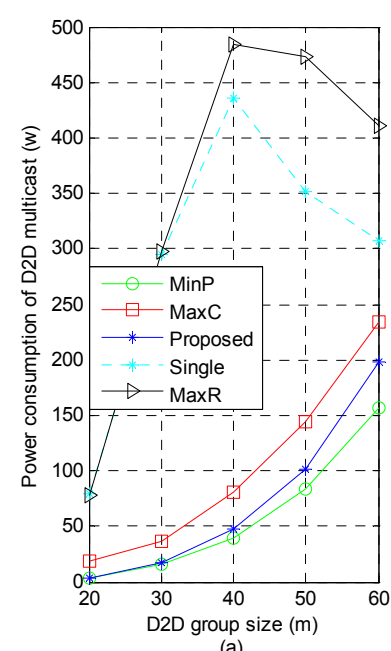

(a)

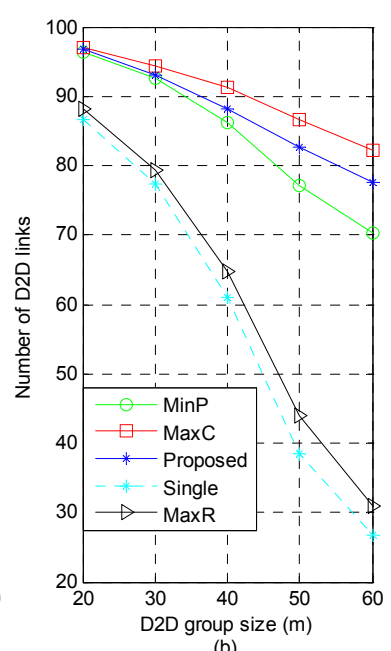

(b)

Figure 3. Power consumption and number of D2D links versus D2D group size: (a) Impact of D2D group size on power consumption; (b) Impact of D2D group size on system capacity. 
The power consumption and system capacity with different cell radius is depicted in Figure 4, where $K=20, M=10,\left|\mathcal{D}_{m}\right|=10, r=50 \mathrm{~m}, \Gamma_{k}^{C}=5 \mathrm{~dB}, \Gamma_{m}^{D}=35 \mathrm{~dB}$ and $\eta=0.5$. Intuitively, the larger the cell radius, the greater the distance between the CUs and D2D receivers in groups, hence the smaller the interference from CUs to the D2D receivers. Thus the number of admitted D2D links increases with the cell radius as shown in Figure $4 \mathrm{~b}$. Similarly, the change in power consumption depends on the following two factors: (i) transmission power decreases along with the lower interference between CUs and the D2D receivers; and (ii) the worst channel condition of D2D multicast becomes relatively worse with the increment of the number of admitted D2D links, thus the required transmission power increases. From Figure 4a, compared to the scheme of reusing single sub-channel ("MaxR" and "Single"), the proposed scheme further utilizes the gain that is brought by the decrement of interference between CUs and the D2D receivers on multiple sub-channels.

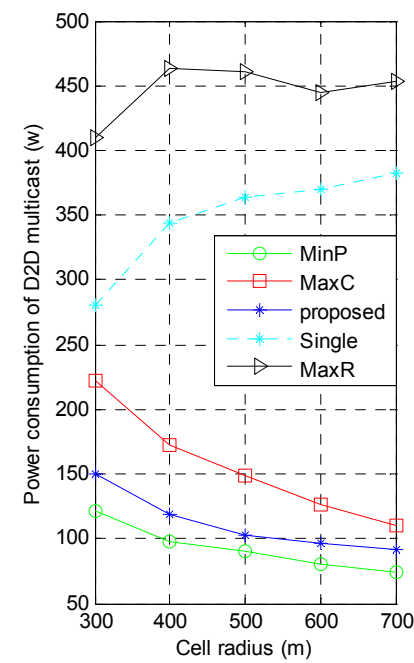

(a)

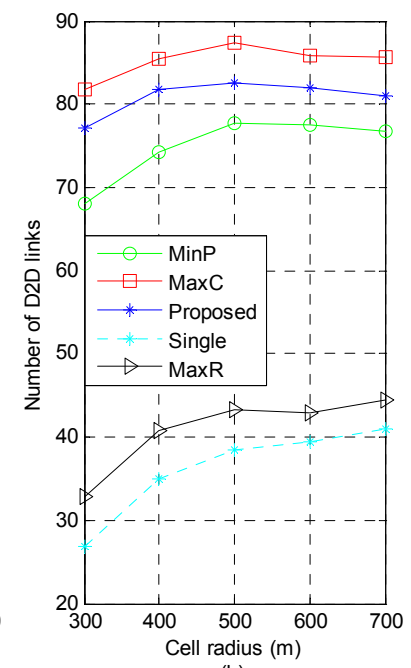

(b)

Figure 4. Power consumption and number of D2D links versus cell radius: (a) Impact of cell radius on power consumption; (b) Impact of cell radius on system capacity.

In Figures 5 and 6 , the power consumption and system capacity performance with different SINR thresholds of CUs and D2D links are illustrated, where $K=20, M=10,\left|\mathcal{D}_{m}\right|=10, R=500 \mathrm{~m}$, $r=50 \mathrm{~m}$ and $\eta=0.5$. It is seen that increasing the SINR threshold leads to decreasing the number of admitted D2D links since it tightens the SINR threshold constraints of both CUs and D2D links and limits the chances for a D2D group to find a CU partner. From Figure 5a, the increase in the SINR threshold of CUs, which has a greater impact on reusing single sub-channel scheme ("MaxR" and "Single"), is helpful to reduce D2D transmission power. As shown in Figure 6a, as the SINR threshold of D2D links increases, the power consumption rises gradually. Furthermore, the power consumption in "MaxR" and "Single" scheme begins a downward trend when the SINR threshold $30 \mathrm{~dB}$. This due to the fact that the dramatic reduction in the number of admitted D2D links reduces the power consumption efficiently.

In Figure 7, the fairness of the proposed scheme is compared with the "MaxR" scheme. The Jain's fairness index is used to evaluate the fairness of the algorithms. The Jain's fairness index is defined as: $f\left(x_{1}, x_{2}, \ldots, x_{M}\right)=\frac{\left(\sum_{i=1}^{M} x_{i}\right)^{2}}{M \cdot \sum_{i=1}^{M} x_{i}^{2}}$ [27], where $x_{i}$ is the resource allocated to D2D groups $i$, and $M$ is the number of D2D groups. In Figure 7, fairness index of two schemes decreases with the increasing number of D2D groups in the network. Nevertheless, the fairness index of the proposed scheme always has higher fairness than referenced scheme since it considers a constrained multi-objective optimization problem. 


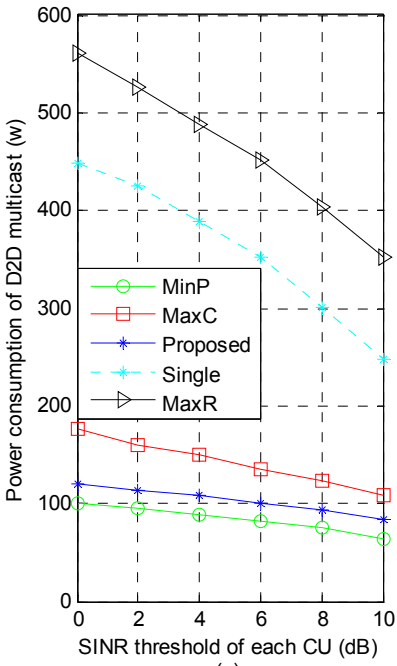

(a)

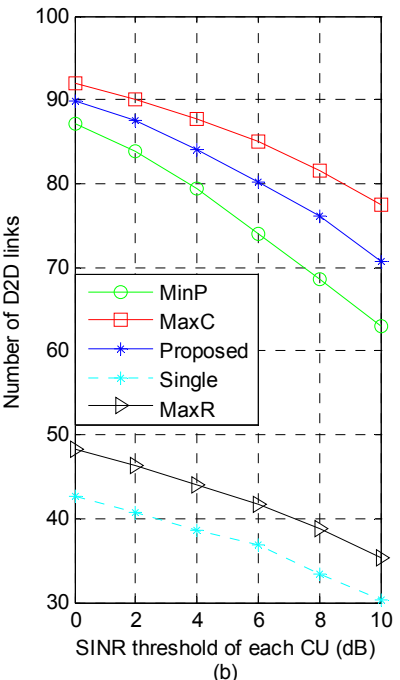

(b)

Figure 5. Power consumption and number of D2D links versus signal-to-interference- plus-noise ratio (SINR) threshold of each CU: (a) Impact of SINR requirement of CUs on power consumption; (b) Impact of SINR requirement of CUs on system capacity.

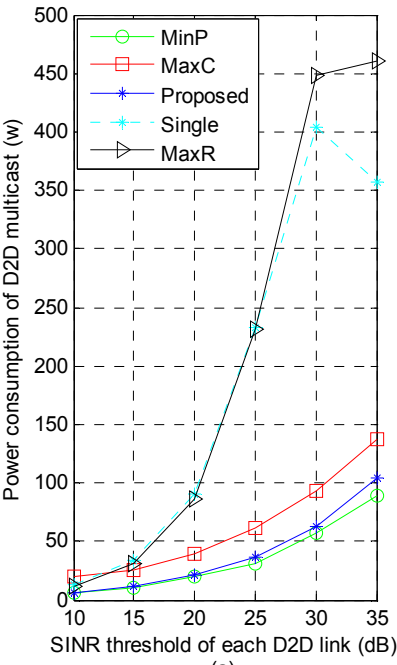

(a)

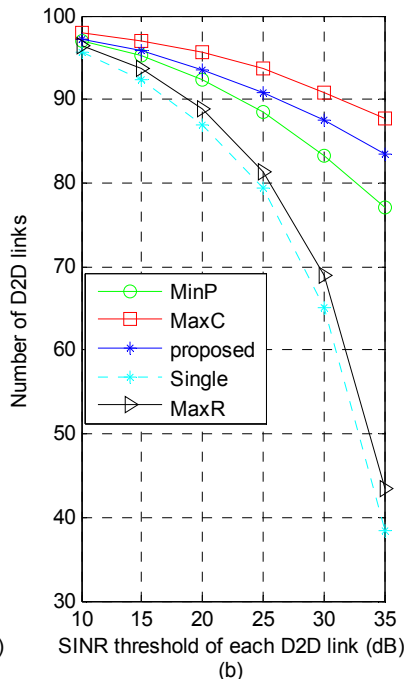

(b)

Figure 6. Power consumption and number of D2D links versus SINR threshold of each D2D link: (a) Impact of SINR requirement of D2D links on power consumption; (b) Impact of SINR requirement of D2D links on system capacity. 

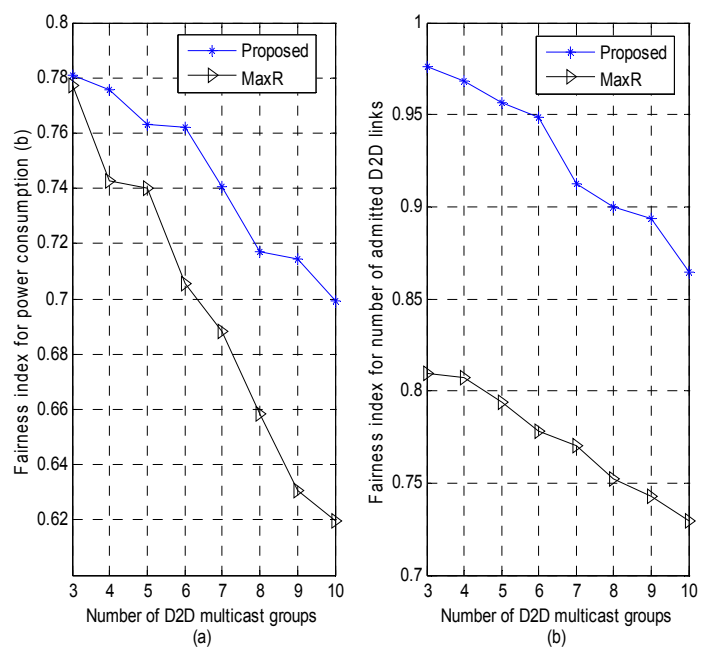

Figure 7. Fairness index versus number of D2D multicast groups: (a) Impact of the number of D2D multicast groups on fairness index for power consumption; (b) Impact of the number of D2D multicast groups on fairness index for system capacity.

\section{Conclusions}

In this paper, a multi-objective resource allocation is studied with QoS guarantees for multiple D2D multicast groups and multiple CUs. Using the linear weighted approach, we formulate a simple but flexible optimization framework in which system power consumption and system capacity are jointly considered. Then, we propose a novel resource allocation scheme that consists of sub-channel allocation and SINR assignment to solve a MINLP problem effectively. Simulation results demonstrate the flexibility and availability of the proposed framework in terms of multi-objective optimization. The results also reveal that the proposed scheme can enhance the performance of power consumption as well as system capacity significantly, and can maintain better fairness for different D2D multicast groups.

Acknowledgments: This work is sponsored by National Natural Science Foundation of China with Grant No. 61373042, and by the Natural Science Foundation of Hubei Province under Grant 2014CFB880.

Author Contributions: Yong Zhang and Fangmin Li initiated and discussed the research problem; Yong Zhang conceived and developed the methods; Yong Zhang and Mohammed Abdulaziz Aide Al-Qaness performed the experiments and made the figures; Yong Zhang and Fangmin Li analyzed the data; and Yong Zhang prepared and wrote the paper.

Conflicts of Interest: The authors declare no conflict of interest.

\section{Appendix A}

By exploiting the features of the constraints $\mathrm{C} 5$ and $\mathrm{C} 6$, the optimal solutions of the SINR values $\gamma_{n, m, k}^{D}$ of the optimization problem in Equation (12) must be the optimal solutions of the following equivalent optimization problem.

$$
\min _{\left\{\gamma_{n, m, k}^{D}\right\}_{m \in \mathcal{M}}} \sum_{k \in \mathcal{S}_{m}} \Phi\left(\gamma_{k, m}^{C *}, \gamma_{n, m, k}^{D}\right)
$$

According to the constraint C7 in Equation (5), if $m \neq m \prime, m \in \mathcal{M}, m \prime \in \mathcal{M}$, then $\mathcal{S}_{m} \cap \mathcal{S}_{m \prime}=\varnothing$. Consequently, the optimization problem in (A1) can be further expressed in the following equivalent $M$ independent optimization problems:

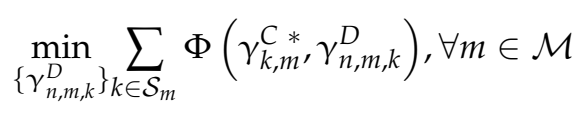

This completes the proof. 


\section{Appendix B}

From Equation (19), $\lambda$ can be expressed as the following function of $\gamma_{n, m, k}^{D}$.

$$
\lambda=D_{k} \cdot \frac{1+\gamma_{n, m, k}^{D}}{\left(H_{k, B}^{C B} H_{m, n}^{D D}-\widetilde{H}_{m, B}^{D B} \widetilde{H}_{k, m, n}^{C D} \Gamma_{k}^{C} \gamma_{n, m, k}^{D}\right)^{2}}
$$

Based on Equation (B1), we can easily prove that $\lambda$ is monotonically decreasing in $\gamma_{n, m, k^{\prime}}^{D}$ as shown in Equation (B2)

$$
\begin{aligned}
\frac{\partial \lambda}{\partial \gamma_{n, m, k}^{D}} & =D_{k} \cdot \frac{H_{k, B}^{C B} H_{m, n}^{D D}+\widetilde{H}_{m, B}^{D B} \widetilde{H}_{k, m, n}^{C D} \Gamma_{k}^{C} \cdot\left(\gamma_{n, m, k}^{D}+2\right)}{\left(H_{k, B}^{C B} H_{m, n}^{D D}-\widetilde{H}_{m, B}^{D B} \widetilde{H}_{k, m, n}^{C D} \Gamma_{k}^{C} \gamma_{n, m, k}^{D}\right)^{3}} \\
& >0
\end{aligned}
$$

Thus, the value range of $\lambda$ can be obtained by substituting $\gamma_{n, m, k}^{D} \in\left[0, \gamma_{n, m, k, \max }^{D}\right]$ into Equation (B1).

\section{References}

1. 3rd Generation Partnership Project (3GPP). Feasibility Study on Proximity-Based Services (ProSe, 3GPP TR 22.803 V12.2.0). Available online: http://www.3gpp.org/ftp/Specs/archive/22_series/22.803/ (accessed on 16 July 2016).

2. Asadi, A.; Wang, Q.; Mancuso, V. A Survey on Device-to-Device Communication in Cellular Networks. IEEE Commun. Surv. Tutor. 2014, 16, 1801-1819. [CrossRef]

3. Fodor, G.; Dahlman, E.; Mildh, G.; Parkvall, S.; Reider, N.; Miklýs, G.; Turýnyi, Z. Design aspects of network assisted Device-to-Device communications. IEEE Commun. Mag. 2012, 50, 170-177. [CrossRef]

4. Datsika, E.; Antonopoulos, A.; Zorba, N.; Verikoukis, C. Green Cooperative Device-to-Device Communication: A Social-Aware Perspective. IEEE Access 2016, 4, 3697-3707. [CrossRef]

5. Xu, S.; Wang, H.; Chen, T.; Huang, Q.; Peng, T. Effective Interference Cancellation Scheme for Device-to-Device Communication Underlaying Cellular Networks. In Proceedings of the IEEE 72nd Vehicular Technology Conference Fall (VTC 2010-Fall), Ottawa, ON, Canada, 6-9 September 2010; pp. 1-5.

6. Pei, Y.; Liang, Y.C. Resource Allocation for Device-to-Device Communications Overlaying Two-way Cellular Networks. IEEE Trans. Wirel. Commun. 2013, 12, 3611-3621. [CrossRef]

7. Zhu, F.; Gao, F.; Yao, M.; Zou, H. Joint Information- and Jamming-Beamforming for Physical Layer Security With Full Duplex Base Station. IEEE Trans. Signal. Process. 2014, 62, 6391-6401. [CrossRef]

8. Li, J.C.F.; Lei, M.; Gao, F. Device-to-device (D2D) communication in MU-MIMO cellular networks. In Proceedings of the IEEE Global Communications Conference (GLOBECOM), Anaheim, CA, USA, 3-7 December 2012; pp. 3583-3587.

9. Golrezaei, N.; Dimakis, A.G.; Molisch, A.F. Device-to-device collaboration through distributed storage. In Proceedings of the IEEE Global Communications Conference (GLOBECOM), Anaheim, CA, USA, 3-7 December 2012; pp. 2397-2402.

10. Zulhasnine, M.; Huang, C.; Srinivasan, A. Efficient Resource Allocation for Device-to-Device Communication Underlaying LTE Network. Mater. Chem. Phys. 2010, 117, 359-364.

11. Lee, J.; Gu, J.; Bae, S.J.; Chung, M.Y. A resource allocation scheme for improving user fairness in device-to-device communication based on cellular networks. In Proceedings of the 7th International Conference on Ubiquitous Information Management and Communication, Kota Kinabalu, Malaysia, 17-19 January 2013; p. 112.

12. Zhao, W.; Wang, S. Low complexity power allocation for device-to-device communication underlaying cellular networks. In Proceedings of the IEEE International Conference on Communications (ICC), Sydney, Australia, 10-14 June 2014; pp. 5532-5537.

13. Hoang, T.D.; Long, B.L.; Le-Ngoc, T. Resource allocation for D2D communications under proportional fairness. In Proceedings of the IEEE Global Communications Conference (GLOBECOM), San Diego, CA, USA, 6-10 December 2015; pp. 595-596. 
14. Wu, D.; Wang, J.; Hu, R.Q.; Cai, Y.; Zhou, L. Energy-Efficient Resource Sharing for Mobile Device-to-Device Multimedia Communications. IEEE Trans. Veh. Technol. 2014, 63, 2093-2103. [CrossRef]

15. Antonopoulos, A.; Kartsakli, E.; Verikoukis, C. Game theoretic D2D content dissemination in 4g cellular networks. IEEE Commun. Mag. 2014, 52, 125-132. [CrossRef]

16. Antonopoulos, A.; Verikoukis, C. Multi-player game theoretic mac strategies for energy efficient data dissemination. IEEE Trans. Wirel. Commun. 2013, 13, 592-603. [CrossRef]

17. Wang, D.; Wang, X.; Zhao, Y. An Interference Coordination Scheme for Device-to-Device Multicast in Cellular Networks. In Proceedings of the IEEE Vehicular Technology Conference (VTC Fall), Québec City, QC, Canada, 3-6 September 2012; pp. 1-5.

18. Yaacoub, E.; Kubbar, O. Energy-efficient Device-to-Device communications in LTE public safety networks. In Proceedings of the IEEE Globecom Workshops (GC Wkshps), Anaheim, CA, USA, 3-7 December 2012; pp. 391-395.

19. Peng, B.; Peng, T.; Liu, Z.; Yang, Y.; Hu, C. Cluster-Based Multicast Transmission for Device-to-Device (D2D) Communication. In Proceedings of the IEEE Vehicular Technology Conference (VTC Fall), Las Vegas, NV, USA, 2-5 September 2013; pp. 1-5.

20. Meshgi, H.; Zhao, D.; Zheng, R. Joint channel and power allocation in underlay multicast device-to-device communications. In Proceedings of the IEEE International Conference on Communications (ICC), London, UK, 8-12 June 2015; pp. 2937-2942.

21. Ng, D.W.K.; Lo, E.S.; Schober, R. Multiobjective resource allocation for secure communication in cognitive radio networks with wireless information and power transfer. IEEE Trans. Veh. Technol. 2014, 65, 3166-3184. [CrossRef]

22. Bousia, A.; Kartsakli, E.; Antonopoulos, A.; Alonso, L. Multiobjective auction-based switching off scheme in heterogeneous networks to bid or not to bid? IEEE Trans. Veh. Technol. 2016. [CrossRef]

23. Doppler, K.; Rinne, M.P.; Janis, P.; Ribeiro, C.; Hugl, K. Device-to-Device Communications; Functional Prospects for LTE-Advanced Networks. In Proceedings of the IEEE International Conference on Communications Workshops, Dresden, Germany, 14-18 June 2009; pp. 1-6.

24. Boyd, S.; Vandenberghe, L. Convex Optimization; Cambridge University Press: New York, NY, USA, 2004.

25. Kaufman, B.; Aazhang, B. Cellular Networks with an Overlaid Device to Device Network. In Proceedings of the 42nd Asilomar Conference on Signals, Systems and Computers, Pacific Grove, CA, USA, 2008; pp. 1537-1541.

26. Jung, M.; Hwang, K.; Choi, S. Joint Mode Selection and Power Allocation Scheme for Power-Efficient Device-to-Device (D2D) Communication. In Proceedings of the IEEE 75th Vehicular Technology Conference (VTC Spring), Yokohama, Japan, 6-9 May 2012; Volume 329, pp. 1-5.

27. Jain, R.; Chiu, D.; Hawe, W. A Quantitative Measure of Fairness and Discrimination for Resource Allocation in Shared Computer Systems. Available online: https://www.researchgate.net/profile/Raj_Jain3/publication/ 220486705_A_Quantitative_Measure_Of_Fairness_And_Discrimination_For_Resource_Allocation_In_Shared_ Computer_Systems/links/09e4150c0274cec7b2000000.pdf (accessed on 7 August 2016).

(c) 2016 by the authors; licensee MDPI, Basel, Switzerland. This article is an open access article distributed under the terms and conditions of the Creative Commons Attribution (CC-BY) license (http://creativecommons.org/licenses/by/4.0/). 